\title{
Theoretical foundations of increasing the efficiency of wide-angle diffusers and practical results of new solutions to this problem
}

\author{
Arkadiy Zaryankin ${ }^{1}$, Mikhail Cherkasov ${ }^{1 *}$, and Ivan Lavyrev ${ }^{1}$ \\ ${ }^{1}$ National Research University "Moscow Power Engineering Institute, Institute of power engineering \\ and mechanic, Department of steam and gas turbines, Krasnokazarmennaya 14, 111250 Moscow, \\ Russian Federation
}

\begin{abstract}
It is shown theoretically that the efficiency of wide-angle diffusers mainly depends on the method of supplying the working medium to the diffuser channel and on the nature of the interaction of this medium with the subsequent streamlined surface of the diffuser. The first condition is due to those changes in the acting force factors within the wall (boundary layer), which occur during the transition from the inlet confuser channel to the subsequent diffuser, and the second follows from the need to ensure an uninterrupted flow of the working medium moving against the increasing (in the direction of travel) static pressure. The above computational studies of wide-angle diffusers have shown that, subject to theoretically justified conditions, it is possible to ensure uninterrupted flow in flat and conical diffusers with an increase in the opening angle $\alpha$ of their flow path to $20^{\circ}$ and with the same degrees of expansion ratio $\mathrm{n}$ to obtain pressure recovery coefficients commensurate with those in diffusers with an angle $\alpha=7^{\circ}$.
\end{abstract}

\section{Introduction}

Conical and annular diffusers with a straight axis are structurally the simplest, but very effective, devices that convert the kinetic energy of moving liquid and gaseous media into potential energy, determined by the value of static pressure in these media.

However, the final effect of such energy conversion depends on a number of additional factors, among which, naturally, the first place is occupied by the geometric parameters of the diffusers. With regard to conical and flat diffusers, such parameters are (see Fig. 1): $\alpha$ opening angle of the flow path; $n=\frac{F_{2}}{F_{1}}$ - the expansion ratio of the channel, where $F_{1}$ is the area of the inlet section, and $F_{2}$ is the area of the outlet section); $\bar{L}=\frac{L}{D_{1}}-$ relative length (for flat diffusers $\bar{L}=\frac{L}{H_{1}}$ ), where $\mathrm{D}_{1}$ and $\mathrm{D}_{2}$ are the diameter of the inlet and outlet sections of the conical diffuser and $\mathrm{H}_{1}$ and $\mathrm{H}_{2}$ - are the heights of the inlet and outlet sections.

All three parameters are related by obvious relationships:

* Corresponding author: mikhail.cherkasov95@yandex.ru 


$$
\begin{gathered}
\sqrt{n}=1+\frac{L}{D_{1}} \operatorname{tg} \frac{\alpha}{2} \text { for conical diffusers } \\
n=1+\frac{L}{h} \operatorname{tg} \frac{\alpha}{2} \text { for flat diffusers. }
\end{gathered}
$$
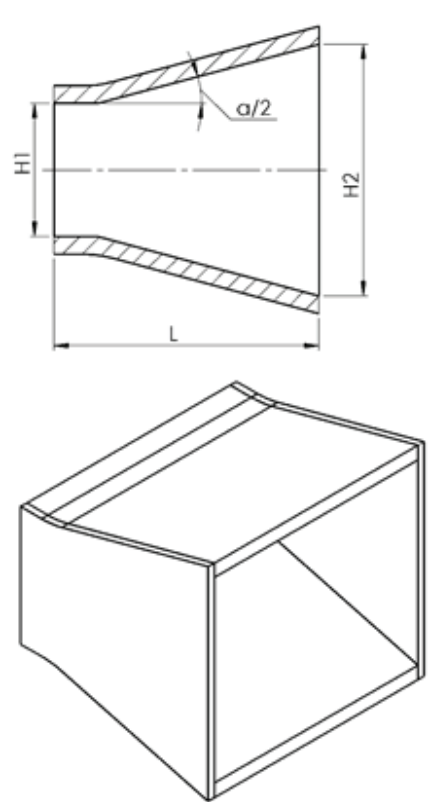

a)
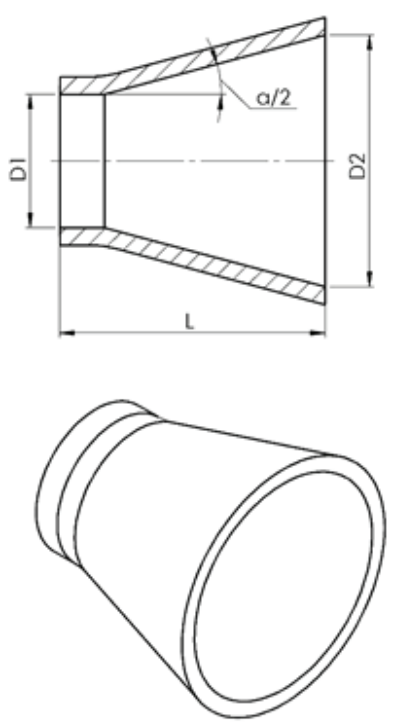

b)

Fig. 1. Types of wide angle diffusers a) - flat diffuser; b) - conical diffuser

From the point of view of obtaining the maximum possible pressure increase in diffusers, the main parameter is the expansion ratio $\mathrm{n}$, an increase in which theoretically leads to an incorrect increase in their efficiency.

However, with a fixed degree of expansion ratio $n$, the degree of pressure recovery (increase in potential energy) in diffusers is decisively influenced by the conditions for the formation of the velocity field when the flow enters the devices under consideration.

In flat and conical diffusers, these conditions largely depend on the opening angle of their flow path.

To illustrate the noted influence on Fig. 2 shows how the coefficient of total energy losses in conical diffusers changes from an increase in the expansion ratio at different values of the opening angle $\alpha[1]$. 


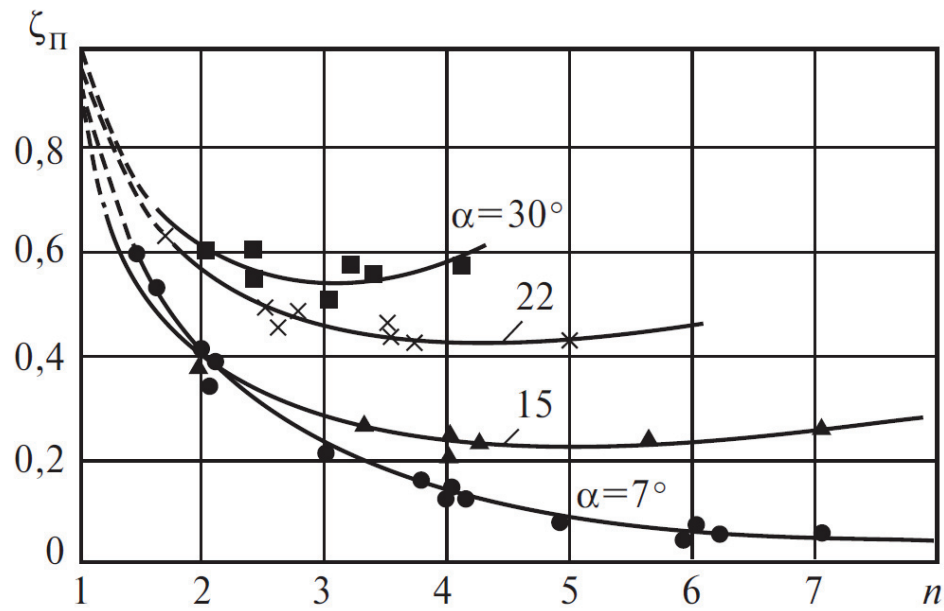

Fig. 2. Influence of the degree of expansion ratio of the conical diffuser $n$ on the coefficient of total energy loss at $\alpha=$ const [1]

It is clearly seen here that only at the angle $\alpha=7^{\circ}$ there is a continuous decrease in the indicated coefficient.

For all values of the angle $\alpha$ exceeding $10^{\circ}$, the dependence $\zeta \mathrm{p}=\mathrm{f}(\mathrm{n}, \alpha)$ has clearly defined values of the coefficient $\zeta$ p, at which this value reaches its minimum value. A further increase in the angle $\alpha$ leads to an increase in losses and, accordingly, to a decrease in the efficiency of the diffusers, due to the emerging separation of the flow from the walls of the diffuser channel.

Thus, the problem of increasing the efficiency of diffusers is reduced to solving theoretical problems associated, firstly, with the physical picture of the flow during the transition from the converging flow in the inlet part of the diffuser to the subsequent diffuser flow, and, secondly, to find a simple and effective method of elimination, or at least would be to the displacement of the section of separation of the flow from the streamlined surfaces in the direction of the outlet section of the diffuser.

Consider the theoretical and practical ways of solving the indicated tasks.

\section{Theoretical foundations for the practical implementation of optimal conditions for the inlet of working media in flat and conical diffusers}

The flow path of classical diffusers shown in Fig. 1 includes an inlet section designed to form a relatively uniform inlet velocity profile at the entrance to the diffuser, the magnitude of the irregularity of which significantly affects the nature of the flow in the subsequent diffuser channel. While maintaining a continuous flow and a fixed back pressure behind the diffuser P2 with an increase in the degree of expansion ratio of the channel n, the pressure P1 in the inlet section continuously decreases, which entails a continuous increase in the pressure recovery factor

$$
\xi\left(\xi=\left(P_{2}-P_{1}\right) /\left(\frac{\rho c_{1}^{2}}{2}\right)\right) .
$$

However, as already indicated in the introduction, in practice such a process is realized only at small opening angles of the flow path of the diffusers, not exceeding $10^{\circ}$. 
With an increase in the angle $\alpha$ over $10^{\circ}$, in the absence of additional measures of external influence on the nature of the flow in the diffuser channel, flow separation from the walls occurs (almost always one-sided). Downstream of the section where flow separation occurred, a pressure close to the pressure $\mathrm{P}_{2}$ behind the diffuser is established, and, accordingly, further pressure recovery does not occur, but an increase in energy losses takes place.

According to the data shown in Fig. 2, with an increase in the angle $\alpha$, the section where the flow separation occurs (the minimum of the total loss coefficient in Fig. 2) shifts towards the inlet section of the diffuser and at $\alpha=30^{\circ}$ the flow separation occurs practically in the inlet section, and the change in the total loss coefficient $\zeta \mathrm{p}$ with an increase in the expansion ratio $\mathrm{n}$ corresponds to a similar dependence in a channel with a sudden expansion ratio of its transverse area.

The experimental data presented clearly indicate that it is the conditions of the flow entering the diffuser that determine the entire subsequent process of converting the kinetic energy of the flow into potential energy determined by the increase in static pressure when the flow of working media moves to the outlet section of the diffuser. In order to understand the physical picture of the change in the initial uniform velocity field when it approaches the inlet section of the diffuser, we present a standard inlet section and point out the reasons that occur during the transition from confuser to diffuser flow (see Fig. 3).

Purely structurally, such a transition most often occurs as a result of a simple attachment of the diffuser itself to the inlet part of the supply confuser. As a result, an angular bend of the streamlined surface occurs in the inlet section 1-1 (see Fig. 3). When flowing around such a surface devoid of an ideal fluid of friction forces, any of its angular kinks is a singular point where the velocity breaks.

In a real liquid, such a velocity discontinuity, of course, is absent, but the indicated angular bend is a source of strong disturbance leading to a local increase in velocity from the side of the confuser supply of the liquid to the diffuser and its no less intense local decrease at the inlet section of the diffuser.

As a result, at the inlet section of the diffuser, the nature of the change in the longitudinal velocity near the streamlined surface, as shown in Fig. 3 turns out to be significantly different from the average flow rate.

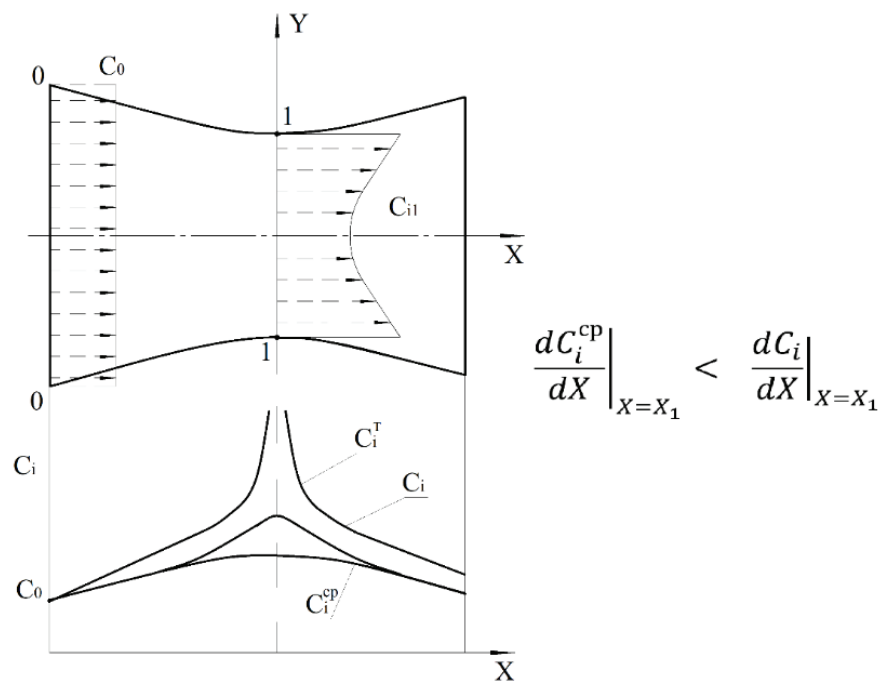

Fig. 3. Dependence of the longitudinal velocity near the streamlined surface of the diffuser 
A sharp increase in the local velocity in the near-wall region of the inlet section 1-1 from the side of the confuser channel with its subsequent no less sharp deceleration at the inlet part of the diffuser itself changes the entire velocity field in section 1-1 (see Fig. 3) and has a very strong effect on on the subsequent flow of the working medium in the subsequent channel.

The velocity field in the inlet section 1-1 of the diffuser takes the form of an "inverted" parabola in the meridial section with apex on the longitudinal axis of the channel under consideration (see Fig. 3).

With such a velocity field, there is a continuous increase in the transverse velocity gradient $\frac{d C_{i}}{d y}$ from the center of the channel to the periphery. An inevitable consequence of such a velocity profile is an increase in the indicated direction and shear stresses $\tau$ proportional to the transverse velocity gradient squared in a turbulent flow regime.

Thus, if, with a uniform (gradientless) velocity field in the transverse direction, the viscosity is limited by the boundary layer region, then in the presence of a flat conical or annular diffuser at the entrance to a flat conical or annular diffuser, the effect of the viscous properties of real liquid and gaseous media covers the entire cross section of the channel. In this case, in accordance with the energy equation at the inlet section of the diffuser in its cross sections, the pressure field also turned out to be nonuniform.

In addition, the fact of a sharp change in the local gradients of velocities and pressures in the region of the angular bend of the streamlined surface is very important (see Fig. 3). When approaching the corner point, the negative value of the local longitudinal pressure gradient near the wall sharply increases, followed by an equally sharp increase in the positive pressure gradient immediately after the flow inlet into the diffuser.

Accordingly, the local positive pressure gradient at the entrance to the diffuser turns out to be significantly higher than the average static value of this value, which significantly increases the probability of flow separation immediately at the entrance to the diffuser.

All the negative factors noted increase both with an increase in speed and with an increase in the opening of the diffuser, which contributes to an earlier separation of the flow from the streamlined surfaces.

It should also be noted that the velocity strongly varying at the inlet section of the diffusers, increasing from the wall to the center of the channel, excludes the possibility of determining the average pressure P1 in the inlet section 1-1 (see Fig. 3), since the local pressure measured on the wall of the inlet section, turns out to be significantly lower than the average flow pressure in this section.

In view of the foregoing, an obvious solution to reduce the unevenness of the velocity profile when the flow enters the diffuser is the elimination of the angular bend of the surface in this section by smooth radial conjugation of the converging inlet section with the subsequent diffuser.

The larger the junction radius, the smaller the difference between the local pressure on the wall and the pressure averaged over the flow rate in the inlet section of the diffuser, and the smaller the inlet positive pressure gradient. Accordingly, one should expect a lower probability of flow separation directly at the flow entry into the diffuser.

However, as studies in [2] have shown, this conclusion turns out to be true only partially.

Smooth flow entry into the diffuser really eliminates flow separation, but at diffuser opening angles above $10^{\circ}$, local flow separation in the inlet section nevertheless occurs. In fig. 4 shows a diagram of a setup for studying diffusers, where pressure oscillograms were taken in a flat diffuser with an opening angle $\alpha=15^{\circ}$ along its longitudinal axis using lowinertia pressure sensors [2]. 


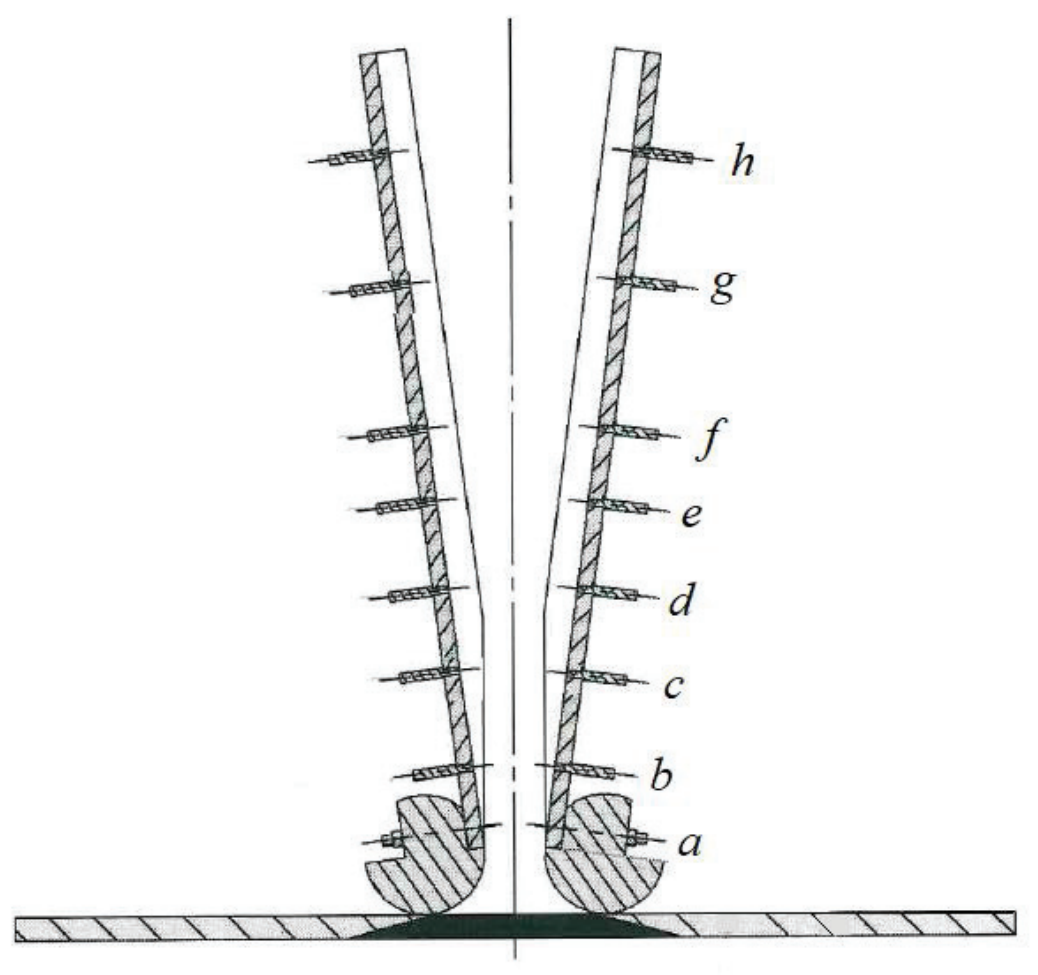

Fig. 4. Installation diagram of pressure sensors in a flat diffuser channel $\alpha=15^{\circ}$ [2]

The results of these measurements, shown in Fig. 5 clearly show the presence of maximum amplitudes of pulsations just after the inlet section of the diffuser, followed by a decrease in pressure fluctuations towards the outlet section.

This result requires a deeper analysis, which is given below. Its essence is based on L. Prandtl's averaged equation for a flat boundary layer:

$$
\rho\left(u \frac{\partial u}{\partial x}+\vartheta \frac{\partial u}{\partial y}\right)=-\frac{d P}{d x}+\frac{d \tau}{d y}
$$

Where $\rho$ - is the flux density; $u, \vartheta$ - velocity components in the Cartesian coordinate system; $\mathrm{P}$ - is the static pressure; $\tau=\tau_{\mathrm{p}}+\tau_{\mathrm{t}}$ is the shear stress in the boundary layer, equal to the sum of the shear stress caused by molecular friction $\left(\tau_{\mathrm{p}}\right)$ and the shear stress $\left(\tau_{\mathrm{t}}\right)$ caused by turbulence within the boundary layer. 

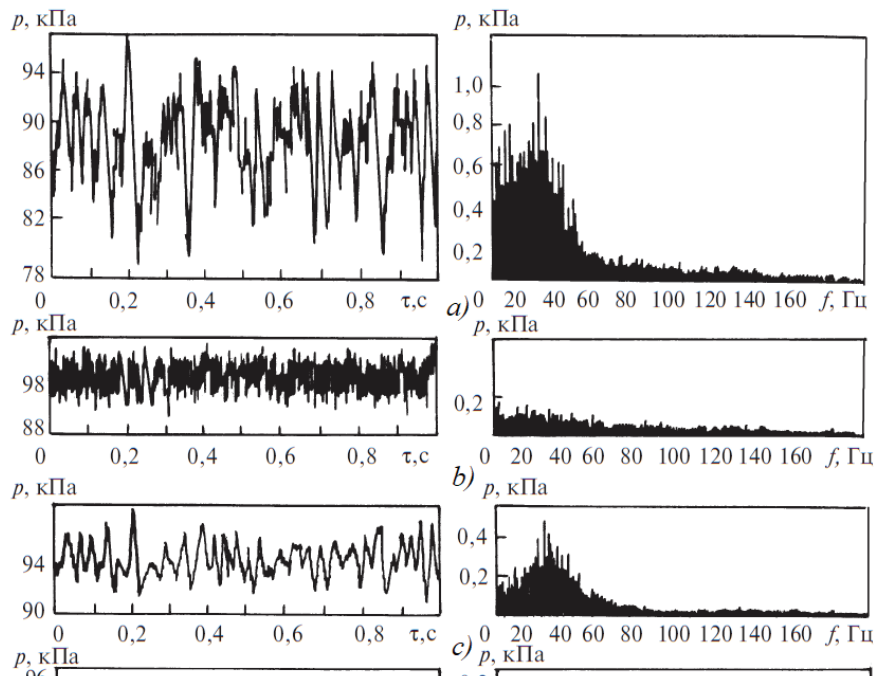

b) $p$, кПа
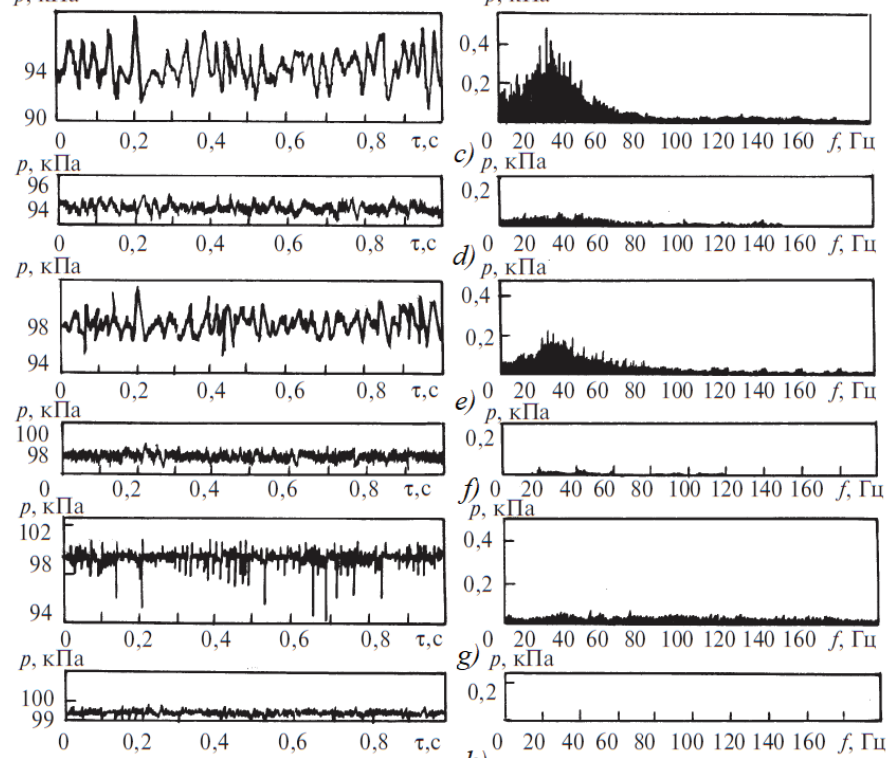

$p, \mathrm{k} \Pi \mathrm{a}$

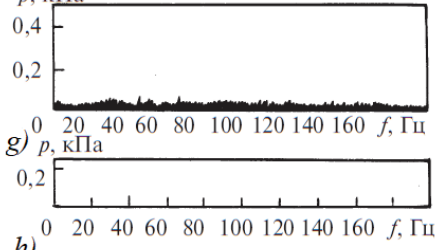

Fig. 5. Oscillogram of pressure pulsations on the wall of a flat diffuser [2]

For further analysis, this fact is very important that directly on the streamlined surface, where $\mathrm{u}=\vartheta=0$, from equation (1) it follows:

$$
\frac{d P}{d x}=\frac{d \tau}{d y}
$$

That is, the sign of the transverse stress gradient in the boundary layer on a solid surface is always equal to the sign of the longitudinal pressure gradient.

Further, it should be noted that equations (1) is the balance equation of the force factors acting within the boundary layer.

Indeed, when multiplying all the terms of equation (1) by the elementary volume of liquid $\mathrm{dV}=\mathrm{dx} \cdot \mathrm{dy} \cdot \mathrm{dz}$, it turns out that the force due to the acting longitudinal pressure gradient $\mathrm{dF}_{\mathrm{p}}$ $=\mathrm{dP} \cdot \mathrm{dy} \cdot \mathrm{dz}$ is equalized by the inertial forces $\mathrm{dF}_{\text {ин }}=\rho \cdot \mathrm{dx} \cdot \mathrm{dy} \cdot \mathrm{dz}\left(u \frac{\partial u}{\partial x}+\vartheta \frac{\partial u}{\partial y}\right)$ and forces due to the gradient of the friction stress $\mathrm{dF}_{\tau}=\mathrm{d} \tau \cdot \mathrm{dx} \cdot \mathrm{dz}$. 
As a result, the balance equation of the force factors acting within the boundary layer takes the following form:

$$
d F_{p}=d F_{\tau}=d F_{\text {in }}
$$

In this record, on the left, there is an external force action on a moving fluid, determined in this case by the geometry of the channel, and on the right side is the response of the flow to an external action that balances the magnitude of the specified action. As long as the above ratio of the force factors is fulfilled, the continuous flow in any channel is also maintained.

It is also important to note one more circumstance, which is that according to the second equation of L. Prandtl for the boundary layer, the transverse pressure gradient within it does not change in the direction of the y-axis. That is, $\frac{d P}{d y}$ in a fixed section of the channel remains constant and, therefore, in any cross section $\frac{d P}{d y}=0$, and, therefore,

$$
d F_{p}=\text { const }=d F_{\tau}-d F_{\text {in }}
$$

From relation (4) follows a direct connection between the nature of the change in the velocity profile within the boundary layer with the profile of the change in the friction stress in this layer.

Based on the boundary conditions on the streamlined surface, where $\frac{d P}{d x}=\frac{d \tau}{d y}$, and on the outer boundary of the boundary layer, $\tau=0$, we construct a qualitative picture of the change in shear stresses during the transition from confuser to diffuser flow (see Fig. 6).

Using the ones shown in Fig. 6 diagrams of the distribution of shear stresses, we will construct the same diagrams for the transverse gradients of friction stresses (see Fig. 7).

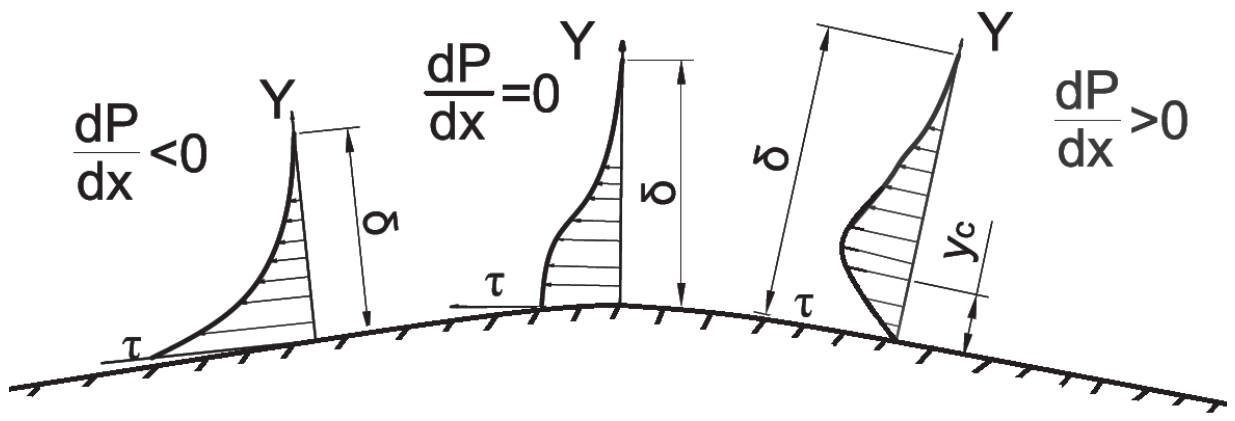

Fig. 6. Diagrams of changes in shear stresses during the transition from converging to diffuser flow 


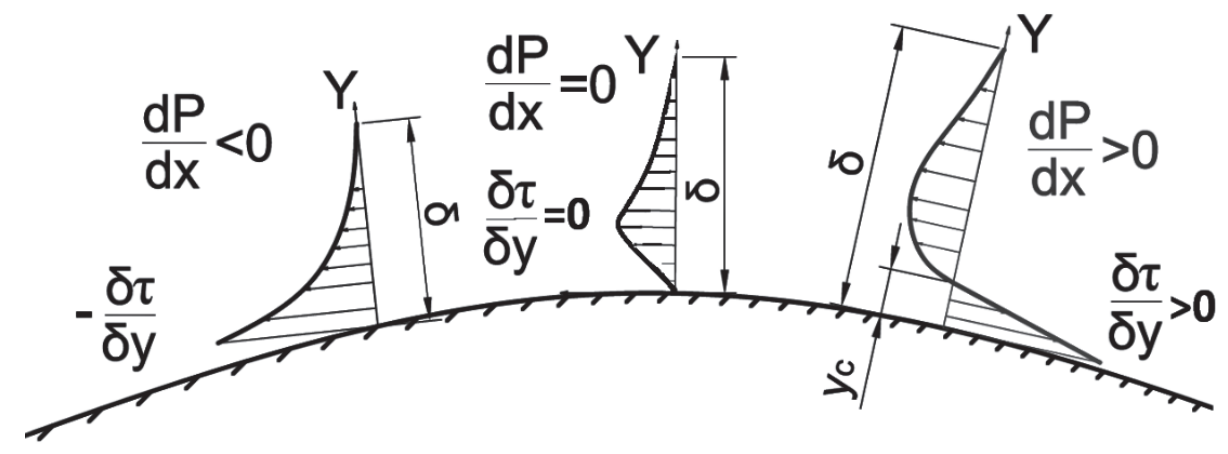

Fig. 7. Diagrams for transverse gradients of friction stresses

The presented distributions of transverse gradients of friction stresses $\frac{d \tau}{d y}=f(y)$ during the transition from confusor flow to diffuser flow show that at a relatively small linear distance, a fundamental change in the distribution pattern of the considered gradients of friction stresses occurs.

If in the confuser flow region (the inlet part of the fluid supply to the diffuser) the shear stresses act throughout the entire thickness of the boundary layer in the direction opposite to the fluid motion, then immediately after the flow enters the diffuser, an almost critical restructuring of the distribution of transverse gradients of tangential stresses and, consequently, forces occurs. caused by these stresses.

Near the streamlined surface at a certain distance yc from the wall, the transverse gradients of tangential stresses change their sign and begin to act in the direction of fluid movement, providing the possibility of fluid movement in the near-wall region against the increasing pressure in the diffuser channel.

In a real flow with inertia, such a restructuring of the acting forces at small linear distances cannot occur, which violates the above balance equation (4), and in practice leads to a local separation of the flow when the liquid enters the diffuser channel even with smooth coupling of the converging channel with diffuser channel (see Fig. 5).

The considered picture of the redistribution of force factors acting within the boundary layer during the transition from confuser to diffuser flow, for the first time theoretically substantiated the need to introduce a cylindrical section in diffusers after the inlet confuser, which then smoothly mates with the diffuser [3].

Note that constructively such a solution was used in the diffuser seats of all new control valves considered in [3].

Our laboratory studies of flat diffusers have shown that the changes made to the scheme of organizing the supply of working media to such diffusers really make it possible to eliminate the local flow separation at the entrance to the diffuser and sharply reduce the amplitude of pressure pulsations in this area. To illustrate what has been said in Fig. 8 shows two oscillograms of pressure pulsations at the inlet to a flat diffuser. 


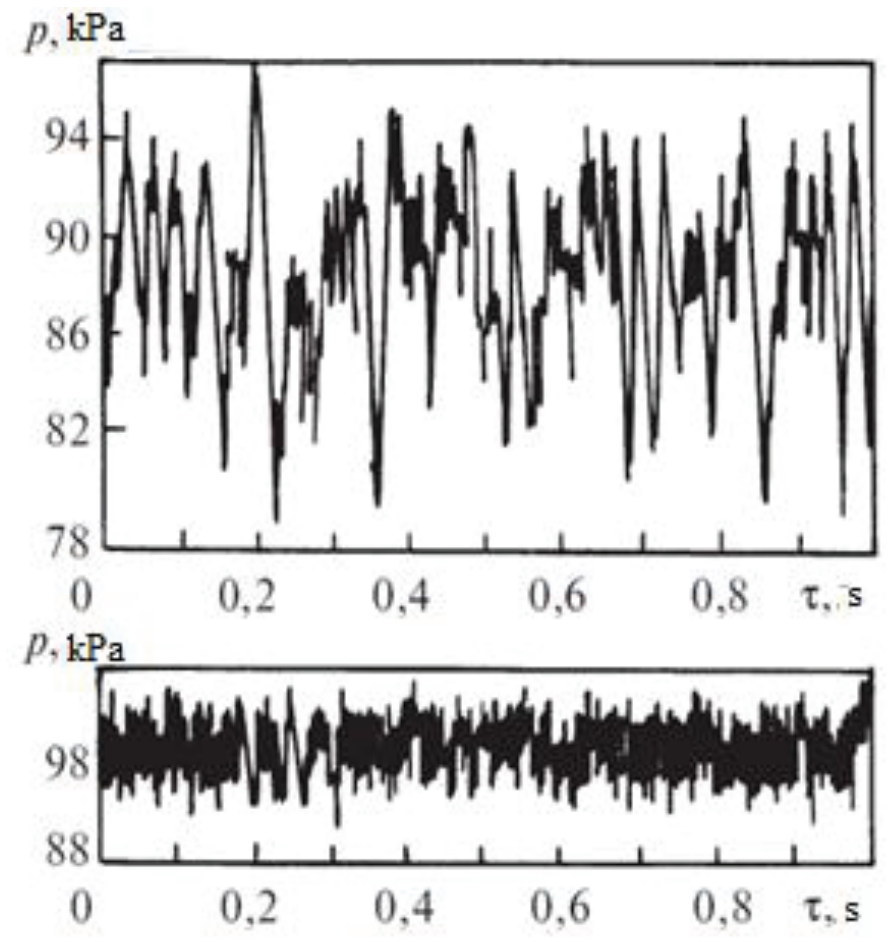

Fig. 8. Oscillograms of pressure pulsations at the inlet to a flat diffuser [2]

The first of them refers to the standard system for supplying air to the diffuser, and the second in the presence of a plane-parallel channel between the inlet confuser and the subsequent diffuser.

However, such structural changes, having reduced the level of dynamic loads on the walls of the diffusers, do not appreciably change the nature of the flow in their subsequent sections and, accordingly, do not lead to an expansion ratio of the maximum permissible expansion ratio angles $\alpha$, which ensure uninterrupted flow in the considered devices.

To solve this main problem, a variety of geometric and aerodynamic methods of external influence on liquid and gaseous media moving in diffusers are used [1].

Among them, the most promising method for maintaining a continuous flow in wideangle diffusers is a method based on changing the conditions of interaction of moving liquid and gaseous media with streamlined surfaces.

So in works $[4,5]$, by passing in flat and conical diffusers from smooth streamlined surfaces to longitudinally ribbed surfaces, it was possible to increase the limiting values of the expansion ratio angle of the flow parts of these diffusers up to $15^{\circ}$ with a simultaneous decrease in several times (at an angle of $15^{\circ}$ in 3, 5 times) vibration accelerations on their walls $[2,5]$.

Continuing this direction, let us consider the theoretical prerequisites for those changes in the shape of streamlined surfaces that can dramatically change the nature of the flow in wide-angle diffusers. 


\section{Theoretical substantiation of the advisability of using slotted surfaces in the direction of flow of streamlined surfaces}

The basis for solving this problem is the classical logarithmic velocity profile, since it was he who formed the idea of the structure of the turbulent boundary layer.

It is this structure that until now underlies almost all works that consider the physical picture of the interaction of all liquid media with streamlined surfaces.

Consider the extent to which this structure corresponds to reality.

To answer this question, let us return to the origins, on the basis of which the structural diagram of the turbulent boundary layer was formed.

It is based on L. Prandtl's semi-empirical theory of turbulence, which made it possible to structurally establish the relationship between turbulent tangential stresses $\tau$ with the averaged longitudinal velocity $\bar{u}$ (we will omit the line in what follows).

$$
\tau=\rho l^{2}\left(\frac{d u}{d y}\right)^{2}
$$

Where $\rho$ - is the density of the moving liquid medium; 1 is the length of the mixing path, at a distance of which turbulent layers moving chaotically in a turbulent flow retain their momentum.

The above relation was taken as a differential equation that determines the velocity profile in a turbulent boundary layer. When integrating it, two conditions $1=\chi y$ and $\tau=$ const were accepted, which are satisfied only in the immediate vicinity of the streamlined surface.

Nevertheless, the subsequent integration of equation (5) was carried out without specifying the limit of integration. As a result of such integration, a well-known equation was obtained that relates the longitudinal velocity $u$ in a cylindrical pipe with the transverse coordinate $y$.

$$
\frac{u}{\vartheta_{*}}=\frac{1}{\chi} \ln y+C
$$

Where $\vartheta_{*}=\sqrt{\frac{\tau}{\rho}}$, having the dimension of speed, is called "dynamic speed", and $\chi$ is some constant determined experimentally.

As always, the constant of integration $\mathrm{C}$ is determined from the boundary conditions of the problem being solved.

With regard to the velocity profile in a tube with a developed turbulent flow or in the boundary layer, the velocity u on the streamlined surface, due to the "sticking" hypothesis, should be equal to zero. For equation (6), this boundary condition, which is central in fluid dynamics, turned out to be impracticable, since at $y=0$ the velocity u tends to infinity.

Hence, it quite naturally follows that the real velocity profile in a turbulent flow regime cannot be described by the obtained relation (6).

However, in this case, the hypothesis came to the rescue that turbulence cannot exist in the near-wall layer and a viscous laminar sublayer remains here, where the friction stresses are determined by Newton's formula $\left(\tau=\mu \frac{d u}{d y}\right)[6,7]$.

In this regard, the constant of integration $\mathrm{C}$ in formula (6) should be determined not from the condition on the streamlined surface, but at a certain distance from it, equal to the thickness of the hypothetical laminar sublayer $\mathrm{y}_{1}$ at a velocity $u=u_{1}$.

As a result, using the experimental data of Nikuradze downstream in pipes, the now classical logarithmic velocity profile was obtained: 


$$
\frac{u}{\vartheta_{*}}=2,5 \ln \frac{\vartheta_{*} y}{v}+5,5
$$

In fluid dynamics, this is apparently a natural case when the structure of the flow of a liquid or gas in the boundary layer was obtained not as a result of a direct physical experiment, but as a result of fitting this structure to dependence (6).

The thus obtained two-layer structure of the turbulent boundary layer turned out to be very convenient for the physical explanation of the independence of the drag coefficient on the Reynolds number when the fluid moves in rough pipes $[6,7]$.

At first glance, all textbooks on fluid dynamics give quite logical considerations about the real existence of a laminar (viscous) sublayer, and only A.P. Melnikov in 1956 [8] and L.D. Landau with E.M. Lifshitz in 1966 [9] asserted the turbulent nature of the flow in the entire cross section of the turbulent boundary layer.

This statement was substantiated in more detail in [10], where it was shown that Eq. (6) does not satisfy any of the three boundary conditions at all.

To prove this, we represent the logarithmic velocity profile (6) in the usual fixed coordinates $\frac{u}{u_{t}}=f\left(\frac{y}{\delta}\right)$ :

$$
\frac{u}{u_{t}}=\frac{\vartheta_{*}}{\chi u_{t}}\left(\ln \frac{y}{\delta}+\ln \frac{\vartheta_{*} \delta}{v}+C\right)
$$

At the outer boundary of the boundary layer at $\mathrm{y}=\delta$, the relative velocity $\frac{u}{u_{t}}$ should be equal to 1 , a $\frac{d\left(\frac{u}{u_{t}}\right)}{d y}=0$ (naturally, the boundary conditions follow from the definition of the boundary layer). If we judge by equation (8) at $\frac{y}{\delta}=1$, that is, on the outer boundary of the boundary layer:

$$
\begin{gathered}
\frac{u}{u_{t}}=\frac{\vartheta_{*}}{\chi u_{t}}\left(\ln \frac{\vartheta_{*} \delta}{v}+C\right)>1 \\
\frac{d}{d X}\left(\frac{u}{u_{t}}\right)=\frac{\vartheta_{*}}{\chi u_{t}}>0
\end{gathered}
$$

The results obtained convincingly show that the logarithmic velocity profile does not satisfy any of the three natural boundary conditions, and it cannot be used in the presented form (8) even when creating a hypothesis of a laminar sublayer.

As for the "brilliant" coincidence of Nikuradze's experiments with the logarithmic profile in handicap (8), it was shown in [9] that when using "floating" coordinates that change with a change in the Reynolds number the dimensions of the quadrants where the corresponding velocity profile is located, then in this case the coinciding experimental points generally turn out to be in different sections of the compared velocity profiles.

The situation changes fundamentally if the integration constant is determined not from the condition on the wall, but from the condition on the outer boundary of the boundary layer, where at $\mathrm{y}=\delta ; \mathrm{u}=\mathrm{ut}$. In this case, using relation (8), we obtain:

$$
\mathrm{C}=\frac{u_{t}}{\vartheta_{*}}-\ln \frac{\vartheta_{*} \delta}{v} \rightarrow \frac{u}{u_{t}}=1+\frac{\vartheta_{*}}{u_{t}} \ln \left(\frac{y}{\delta}\right)
$$


The logarithmic velocity profile in the form (9) practically satisfies all three boundary conditions without the need to introduce the concept of a laminar sublayer, since the ratio $\frac{u}{u_{t}}=0$ at a distance from the wall $\frac{y}{\delta}=e^{-\frac{u_{t}}{\vartheta_{*}}}$

Since $\frac{u_{t}}{\vartheta_{*}}$ in Nikuradze's experiments varies within $10 \div 35$, then $\frac{y}{\delta}<0,01 \mathrm{~mm}$.

The analysis shows that it is not a viscous sublayer that actually contacts the streamlined surface, but a turbulent flow with a sufficiently high friction stress, which makes it possible to overcome large positive longitudinal pressure gradients acting in the diffuser.

In support of what has been said, attention should be paid to the fact that a necessary condition for the onset of turbulence is the presence of a shear flow [11], the maximum value of which occurs precisely near the streamlined surface.

Accordingly, it is this region that is not a region of transition from a viscous flow to a subsequent turbulent one within the boundary layer, but a region of intense turbulence generation followed by its attenuation in the direction normal to the streamlined surface [1].

However, in spite of the above considerations about the two-layer structure of the turbulence of the velocity profile, the very idea of such a structure can turn out to be very fruitful if a stable laminar layer is created artificially between the turbulent flow and the streamlined surface, ensuring the contact of the turbulent flow not with a solid surface, but with an artificially created near the wall. a laminar interlayer of a moving liquid or gaseous medium.

Technically, this problem can be solved by passing from smooth streamlined surfaces to slit surfaces in the direction of the flow.

A variant of a flat diffuser with such walls is shown in Fig. 9. Here the streamlined surfaces are made with very narrow longitudinal slots, the width of which does not exceed $b$ $=2 \mathrm{~mm}$ at the channel depth $\mathrm{h}=7-10 \mathrm{~mm}$ and the thickness of the partitions between the slots $\mathrm{a}=0.8-1 \mathrm{~mm}$.

With this design of a streamlined surface in rectangular slots with a large relative depth $\frac{h}{b} \approx 5 \div 6$, due to the large influence of viscosity and small dimensions of the slots, the transition to a turbulent flow cannot occur. As a result, the turbulent flow external to the thin edges will no longer come into contact with a solid surface, where, according to the adhesion hypothesis, the velocity vanishes, but with a laminar flow, the velocity of which on the plane of contact with the external turbulent flow will no longer be zero, but much less than the external velocity flow.

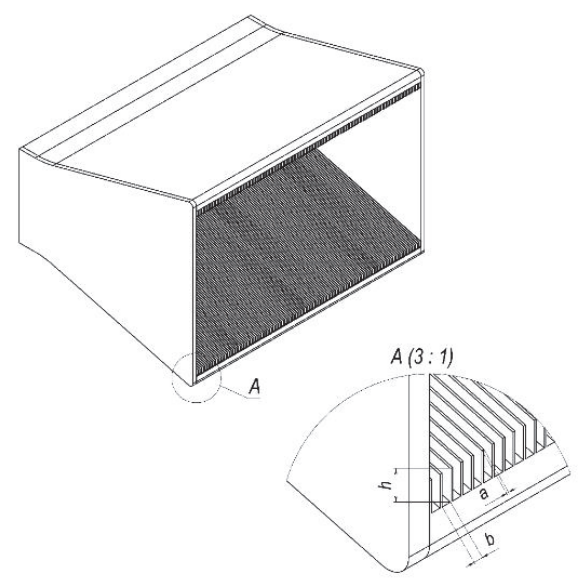

Fig. 9. Flat diffuser with ribbed surfaces 
Such a measure will sharply reduce the transverse velocity gradient at the outer boundary of the ribbed surface and cause additional turbulization of the flow in the region of two flows with different velocities, thereby ensuring the possibility of maintaining an unseparated flow in diffusers with large degrees of expansion ratio n, expansion ratio angles $\alpha$ exceeding $10 \div$ $15^{\circ}$.

\section{Conclusions}

1. As a result of the analysis, the deformation of the velocity profile during the supply of working media to the inlet section of the diffuser showed that this deformation primarily limits the opening angles of the flow paths of flat and conical diffusers, exceeding which causes a local separation of the flow immediately behind the inlet section.

2. It is shown that in order to prevent local flow separation when the flow enters the diffuser between the supplied confuser and the subsequent diffuser, it is necessary to introduce a plane-parallel and cylindrical section, which provides the possibility of restructuring the direction of the acting transverse gradients of friction stresses in the near-wall region from negative to positive values.

3. The analysis of the logarithmic velocity profile in a turbulent boundary layer does not correspond to any of the three natural boundary conditions arising from the definition of the boundary layer, and its two-layer structure, which asserts the existence of a viscous (laminar) sublayer near the walls, is only a consequence of the desire to impart a logarithmic velocity profile physical meaning.

4. Using the hypothesis of a laminar sublayer, it is proposed to artificially create a stable laminar flow near the wall by passing to streamlined slit surfaces, and thus ensuring contact of the main stream not with a solid wall, but with a laminar flow in the interslit space.

\section{References}

1. Zaryankin A.E. Mechanics of incompressible and compressible fluids. Publishing House MPEI 2014.

2. Zaryankin A.E., Gribin V.G., Paramonov A.N., Fichoryak O.M., Noskov V.V. The influence of the opening angle of diffusers on their vibration state and some ways to reduce these vibrations, Teploenergetika 2012, no. 9 p. 27-36.

3. Zaryankin A.E., Simonov B.P. Steam turbine control and one hundred-porn-control valves. Moscow: MPEI Publishing House, 2005.

4. Grigoriev E.Yu. Development and research of ways to reduce vibration of annular diffusers of gas turbines: dis ... cand. tech. Sciences: 05.04.12 / Grigoriev E.Yu. -M .: 2014. - 259 p.

5. Noskov V.V. Experimental study of flow modes on the vibrodynamic state of diffuser elements of the flow parts of turbomachines: dis ... cand. tech. Sciences: 05.04.12 / Noskov V.V. -M .: 2010. - 230 p.

6. Loytsyansky L.G. Mechanics of liquids and gases. Publishing house "Science" 1970.

7. Schlichting G. Theory of the boundary layer. Publishing house "Science" 1969.

8. Melnikov A.P. Fundamentals of Theoretical Aerodynamics. LKVVIA Publishing House 1953.

9. Landau L.D., Lifshits E.M. Continuum mechanics. SHTTL. M. 1954 - 532 p. 
10. Zaryankin A.E. To the question of a logarithmic velocity profile correspondence with the experimental

11. Schubauer G.B., Chen K.M. Turbulent flow and heat transfer / Per. from English // ed. Lin-Jia-Jio. Moscow: Foreign Literature Publishing House. 1963 .- 563 p. 